\title{
GOLDIE DIMENSION, DUAL KRULL DIMENSION AND SUBDIRECT IRREDUCIBILITY
}

\author{
TOMA ALBU \\ 'Simion Stoilow' Institute of Mathematics of the Romanian Academy, P.O. Box 1-764, \\ RO-010145 Bucharest 1, Romania \\ e-mail:Toma.Albu@imar.ro
}

\begin{abstract}
In this survey paper we present some results relating the Goldie dimension, dual Krull dimension and subdirect irreducibility in modules, torsion theories, Grothendieck categories and lattices. Our interest in studying this topic is rooted in a nice module theoretical result of Carl Faith [Commun. Algebra 27 (1999), 1807-1810], characterizing Noetherian modules $M$ by means of the finiteness of the Goldie dimension of all its quotient modules and the ACC on its subdirectly irreducible submodules. Thus, we extend his result in a dual Krull dimension setting and consider its dualization, not only in modules, but also in upper continuous modular lattices, with applications to torsion theories and Grothendieck categories.
\end{abstract}

2000 Mathematics Subject Classification. 16P60, 16S90, 18E15, 06C05, 06B35.

1. Introduction. A lovely 10 years old result of Carl Faith [21, 22] states:

Faith's Theorem (FT). A module is Noetherian if and only if it is QFD and satisfies the ACC on subdirectly irreducible submodules.

Recall that a module $M_{R}$ is called quotient finite dimensional (or $Q F D$ ) [17], if any quotient module of $M$ has finite Goldie (or uniform) dimension. If we denote for a module $M$ by $\mathcal{L}(M)$ the lattice of all its submodules and by $\mathcal{S}(M)$ the subset of $\mathcal{L}(M)$ consisting of all subdirectly irreducible submodules of $M$, then the FT can be stated as follows:

$\mathcal{L}(M)$ is a Noetherian poset $\Longleftrightarrow M$ is QFD and $\mathcal{S}(M)$ is a Noetherian poset.

Now observe that an arbitrary poset $P$ is Noetherian if and only if it has dual Krull dimension $k^{0}(P) \leqslant 0$. Thus, the FT can be reformulated in a dual Krull dimension setting as follows:

$$
\text { FT }_{0}: k^{0}(\mathcal{L}(M)) \leqslant 0 \Longleftrightarrow \mathcal{L}(M) \text { is a QFD lattice and } k^{0}(\mathcal{S}(M)) \leqslant 0 .
$$

The following natural problems related to FT arise:

(1) Investigate whether the dual $\mathbf{F T}^{0}$ of the FT hold.

(2) Do the above reformulation $\mathbf{F T}_{0}$ of the FT hold for an arbitrary ordinal $\alpha$ instead of 0 , i.e. is the following statement

$$
\text { FT }_{\alpha}: k^{0}(\mathcal{L}(M)) \leqslant \alpha \Longleftrightarrow \mathcal{L}(M) \text { is a QFD lattice and } k^{0}(\mathcal{S}(M)) \leqslant \alpha
$$

true? A similar question for its dual $\mathbf{F T}_{\alpha}^{0}$.

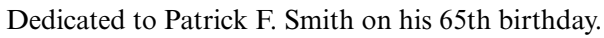


(3) Extend (2) from the lattice $\mathcal{L}(M)$ to an arbitrary upper continuous modular lattice $L$.

(4) Apply (3) to Grothendieck categories and to module categories equipped with hereditary torsion theories.

The aim of this survey paper is to present the answers, we know so far, to these four questions. We will also illustrate here a general strategy, which consists on putting a module-theoretical theorem, in our case the Faith's Theorem, in a latticial frame, in order to translate that theorem to module categories equipped with a hereditary torsion theory and to Grothendieck categories.

2. Subdirectly irreducible modules. The concept of subdirectly irreducible (SI) appears in various circumstances: universal algebras, rings, modules, lattices, posets, etc. Remember that a classical result of Birkhoff [15] states that any universal algebra is a subdirect product of SI algebras.

Loosely speaking, an object of a category with direct products is called subdirectly irreducible if it cannot be represented as a subdirect product of 'smaller' objects (i.e. proper epimorphic images). We shall illustrate below more precisely this concept for module categories.

Throughout this paper $R$ will denote an associative ring with non-zero identity element, and Mod- $R$ the category of all unital right $R$-modules. The notation $M_{R}$ will be used to designate a unital right $R$-module $M$. The lattice of all submodules of a module $M_{R}$ will be denoted by $\mathcal{L}\left(M_{R}\right)$. We denote by $\mathbb{N}$ the set $\{0,1,2, \ldots\}$ of all natural numbers, by $\mathbb{Z}$ the ring of rational integers and by $\mathbb{R}$ the field of real numbers.

A module $M_{R}$ is called subdirectly irreducible if any representation of $M$ as a subdirect product of other modules is trivial, i.e. for every family $\left(M_{i}\right)_{i \in I}$ of right $R$-modules and for every monomorphism $\varepsilon: M \longmapsto \prod_{i \in I} M_{i}$ such that $\pi_{j} \circ \varepsilon$ is an epimorphism $\forall j \in I, \exists i \in I$ such that $\pi_{i} \circ \varepsilon$ is an isomorphism, where $\pi_{j}: \prod_{i \in I} M_{i} \rightarrow M_{j}$, $j \in I$, are the canonical projections. The concept of subdirectly irreducible module turns out to be the dual of that of cyclic module as we will see below.

Clearly, a module $M_{R}$ is cyclic if and only if it satisfies the following condition:

$$
\begin{gathered}
\exists x_{0} \in M, \forall N \in \operatorname{Mod}-R, \forall f \in \operatorname{Hom}_{R}(N, M) \quad \text { with } \quad x_{0} \in \operatorname{Im}(f) \\
\Longrightarrow f \text { is an epimorphism. }
\end{gathered}
$$

Dually, a module $M_{R}$ is said to be cocyclic if it satisfies the following condition:

$$
\begin{gathered}
\exists x_{0} \in M, \forall N \in \operatorname{Mod}-R, \forall g \in \operatorname{Hom}_{R}(M, N) \quad \text { with } \quad x_{0} \notin \operatorname{Ker}(g) \\
\Longrightarrow g \text { is a monomorphism. }
\end{gathered}
$$

To the best of our knowledge, the notion of cocyclic module appears for the first time in the literature in Fuchs [23, Section 3].

The next result (see e.g. $[\mathbf{4 2}, \mathbf{1 4 . 8 ]}$ ) provides various characterizations of cocyclic modules, which will naturally lead below to the most general concept of a subdirectly irreducible poset (see Definition 4.1).

Proposition 2.1. The following statements are equivalent for a non-zero module $M_{R}$

(1) $M$ is cocyclic.

(2) $\bigcap_{0 \neq X \leqslant M} X \neq 0$. 
(3) The poset $\mathcal{L}(M) \backslash\{0\}$, ordered by inclusion, has a least element.

(4) $M$ has a simple essential socle.

(5) $M$ is subdirectly irreducible.

For any module $M_{R}$ we have obviously $M=\sum_{C \in \mathcal{C}(M)} C$, and dually, less obviously $0=\bigcap_{X \in \mathcal{S}(M)} X$, where $\mathcal{C}(M):=\{C \leqslant M \mid C$ is cyclic $\}$ and $\mathcal{S}(M):=$ $\{X \leqslant M \mid M / X$ is cocyclic $\}$.

Note that the elements of $\mathcal{S}(M)$ are called subdirectly irreducible submodules of $M$ in Faith [21, 22]. So, $X \leqslant M$ is a subdirectly irreducible submodule of $M$ if and only if the module $M / X$ is subdirectly irreducible.

3. $\mathbf{F T}^{0}$. In this section we present a statement $\mathbf{F T}^{0}$ dual to that of Faith's Theorem FT, which gives a characterization of Artinian modules $M_{R}$ in terms of submodules of $M$ which behave dually to the submodules in $\mathcal{S}(M)$. Note that Artinian modules are precisely those modules having Krull dimension $\leqslant 0$, hence it seems natural to ask also for similar characterizations of modules having Krull dimension at most a given ordinal $\alpha \geqslant 0$. This Krull dimension setting will be discussed in the subsequent sections.

We will denote by $\mathcal{F}(M)$ the set of all finitely generated submodules of a module $M$.

Theorem 3.1 (The Dual Faith Theorem FT ${ }^{0}$ ) ([8, Theorems 1.12 and 1.13]). The following statements are equivalent for a module $M_{R}$.

(1) $M$ is Artinian.

(2) $M$ is $\mathrm{QFD}$ or $\mathrm{SFD}^{0}$, and $C$ is Artinian for any $C \in \mathcal{C}(M)$.

(3) $M$ is $\mathrm{QFD}$ or $\mathrm{SFD}^{0}$, and $\mathcal{C}(M)$ is an Artinian poset.

(4) $M$ is QFD or $\mathrm{SFD}^{0}$, and $F$ is Artinian for any $F \in \mathcal{F}(M)$.

(5) $M$ is $\mathrm{QFD}$ or $\mathrm{SFD}^{0}$, and $\mathcal{F}(M)$ is an Artinian poset.

Recall that a module $M_{R}$ is called $s u b$ finite dual dimensional (or $S F D^{0}$ ), if any submodule of $M$ has finite dual uniform (or dual Goldie, or hollow) dimension. So, the term of a SFD ${ }^{0}$ module is dual to that of a QFD module. The reader is referred to $[\mathbf{2 7}, \mathbf{3 8}, \mathbf{4 0}]$ for the concept of dual uniform dimension of modules and modular lattices.

As it is well known, any module with Krull (or dual Krull dimension) is QFD, but a module with Krull dimension is not necessarily $\mathrm{SFD}^{0}$ : the Abelian group $\mathbb{Z}$ is a Noetherian $\mathbb{Z}$-module, but it does not have dual Goldie dimension. However, if $M_{R}$ satisfies the property AB5* (this means that the lattice $\mathcal{L}\left(M_{R}\right)$ of all submodules of $M$ is lower continuous), in particular if $M$ is linearly compact, then $M$ is QFD if and only if $M$ is $\mathrm{SFD}^{0}$, by [28, Lemma 6]. Notice that any $\mathrm{SFD}^{0}$ module is QFD, by [41, Proposition 12].

4. Latticial background. For a partially ordered set, shortly poset, $(P, \leqslant)$ and elements $a \leqslant b$ in $P$ we write

$$
\begin{gathered}
b / a:=[a, b]=\{x \in P \mid a \leqslant x \leqslant b\}, \\
{[a, b[:=\{x \in P \mid a \leqslant x<b\},} \\
] a, b]:=\{x \in P \mid a<x \leqslant b\} .
\end{gathered}
$$


All posets considered in this paper are assumed to have a least element denoted by 0 and a last element denoted by 1 . If $x<y$ are elements of a poset $P$ and there is no $z \in P$ such that $x<z<y$, then we say that $x$ is covered by $y$, and we write $x \prec y$. An element $a \in P$ is said to be an atom of $P$ if $0 \prec a$.

We denote by $\mathcal{L}$ (resp. $\mathcal{M}, \mathcal{C}, \mathcal{U}$ ) the class of all lattices with 0 and 1 (resp. modular lattices with 0 and 1 , complete lattices, upper continuous lattices). Throughout this paper a lattice will always mean a member of $\mathcal{L}$, and $(L, \leqslant, \wedge, \vee, 0,1)$, or more simply, just $L$, will always denote such a lattice. The opposite lattice of $L$ will be denoted by $L^{0}$. If $L \in \mathcal{C}$, then for every subset $S$ of $L$ we denote $\wedge S=\bigwedge_{x \in S} x$ and $\bigvee S=\bigvee_{x \in S} x$. An element $e$ of a lattice $L$ is said to be essential in $L$ if $e \wedge x \neq 0$ for each $0 \neq x \in L$. Dually, an element $s \in L$ is small in $L$ if $s$ is essential in $L^{0}$, i.e. if $s \vee x \neq 1$ for every $x \neq 1$ in $L$. If $L \in \mathcal{C}$, then the socle $\operatorname{Soc}(L)$ of $L$ is the join of all atoms of $L$. A lattice $L$ is said to be semi-Artinian if for any $1 \neq x \in L$, the lattice $1 / x$ has at least an atom. An element $c$ of a lattice $L \in \mathcal{C}$ is compact in $L$ if whenever $c \leqslant \bigvee_{x \in A} x$ for a subset $A$ of $L$, there is a finite subset $F$ of $A$ such that $c \leqslant \bigvee_{x \in F} x$. The lattice $L$ is compact if 1 is a compact element in $L$, and compactly generated if every element of $L$ is a join of compact elements.

For all undefined notation and terminology on lattices, the reader is referred to $[16,18,26]$ and/or [39].

The next definition is inspired by Proposition 2.1 .

Definition 4.1. A poset $P$ is said to be subdirectly irreducible, abbreviated $S I$, if $P \neq\{0\}$ and the set $P \backslash\{0\}$ has a least element; i.e. there exists an element $0 \neq x_{0} \in P$ such that $x_{0} \leqslant x$ for every $0 \neq x \in P$. An element $s \in P$ is said to be a subdirectly irreducible element of $P$ if the interval $1 / s$ is a subdirectly irreducible poset, and the set of all subdirectly irreducible elements of $P$ will be denoted by $\mathcal{S}(P)$.

Observe that a module $M_{R}$ is subdirectly irreducible if and only if the lattice $\mathcal{L}\left(M_{R}\right)$ of all submodules of $M_{R}$ is subdirectly irreducible, and the poset $\mathcal{S}\left(M_{R}\right)$ defined just after Proposition 2.1 is exactly $\mathcal{S}\left(\mathcal{L}\left(M_{R}\right)\right)$.

Definitions 4.2. (a) A lattice $L$ is said to be co-irreducible or uniform (resp. completely co-irreducible or completely uniform) if $L \neq\{0\}$ and $x \wedge y \neq 0$ for any non-zero elements $x, y \in L$ (resp. $\bigwedge_{i \in I} x_{i} \neq 0$ for any non-empty family $\left(x_{i}\right)_{i \in I}$ of non-zero elements $x_{i} \in L$ ).

(b) An element $x$ of a lattice $L$ is said to be irreducible, (resp. completely irreducible, abbreviated $C I$ ) if $x \neq 1$ and whenever $x=a \wedge b$ for $a, b \in L$, then $x=a$ or $x=b$ (resp. whenever $x=\bigwedge_{i \in I} a_{i}$ for a non-empty family $\left(a_{i}\right)_{i \in I}$ of elements of $L$, then $x=a_{j}$ for some $j \in I$ ).

Clearly, an element $x \in L$ is irreducible (resp. completely irreducible) if and only if the lattice $1 / x$ is co-irreducible (resp. completely co-irreducible). For any lattice $L$ we denote by $\mathcal{I}(L)$ the set of all irreducible elements of $L$, and by $\mathcal{I}^{c}(L)$ the set of all completely irreducible elements of $L$. For any module $M_{R}$, we set $\mathcal{I}\left(M_{R}\right):=\mathcal{I}\left(\mathcal{L}\left(M_{R}\right)\right)$ and $\mathcal{I}^{c}\left(M_{R}\right):=\mathcal{I}^{c}\left(\mathcal{L}\left(M_{R}\right)\right)$.

If $L \in \mathcal{C}$, then clearly $s \in L$ is a subdirectly irreducible element of $L$ if and only if $s$ is completely irreducible, so $\mathcal{S}(L)=\mathcal{I}^{c}(L)$. In the sequel, for the term of subdirectly irreducible element of any lattice, we will occasionally use the more suggestive term of completely irreducible (CI) element.

The next result is a lattice extension of Proposition 2.1. 
Proposition 4.3 ([2, Proposition 0.5]). The following statements are equivalent for a lattice $L \in \mathcal{C}, L \neq\{0\}$.

(1) $L$ is subdirectly irreducible.

(2) $\bigwedge_{x \in L \backslash\{0\}} x \neq 0$.

(3) $L$ is completely co-irreducible.

(4) L has an atom a that is essential in $L$.

(5) $L$ is co-irreducible and $\operatorname{Soc}(L) \neq 0$.

For a poset $P$ we denote by $k(P)$ (resp. $\left.k^{0}(P)\right)$ the Krull dimension (resp. the dual Krull dimension) of $P$ (see also [10, Section 3]). The notation $k(P) \leqslant \alpha$ means that $P$ has Krull dimension, and this is $\leqslant$ than the ordinal $\alpha$. A nice result due to Lemonnier [31, Corollaire 6] states that an arbitrary poset $P$ has Krull dimension if and only if it has dual Krull dimension. Any poset having Krull dimension has also Gabriel dimension, but in general, not conversely.

For the definition and basic properties of the Krull dimension and dual Krull dimension (resp. Gabriel dimension) of a poset the reader is referred to [31] or [33] (resp. to [1] or [35]).

5. QFD lattices. Let $L \in \mathcal{L}$ be a lattice. Recall that a set $S$ of non-zero elements of $L$ is said to be independent if for every finite subset $F$ of $S$ and for each $s \in S \backslash F$, one has $s \wedge\left(\bigvee_{x \in F} x\right)=0$.

Definition 5.1. One says that a lattice $L \in \mathcal{L}$ has finite Goldie (or uniform) dimension if there is no infinite independent subset of $L$. The lattice $L$ is said to have finite dual Goldie dimension if $L^{0}$ has finite Goldie dimension. The lattice $L$ is called $Q F D$ (i.e. quotients have finite Goldie dimension) if $1 / x$ has finite Goldie dimension for every $x \in L$.

The reader is referred to the survey paper [36] in this Proceedings for more about Goldie dimension of modular lattices.

The next result, originally proved for modules by Lemonnier (see [32, Lemme 1.1]), is an important tool for studying the QFD property of upper continuous modular lattices.

LEMMA 5.2 (LemONNIER's LemMA) ([10, Lemma 3.4]). Let $\mathbb{P}$ be a property of the class $\mathcal{M} \cap \mathcal{U}$ of all upper continuous modular lattices, which satisfies the following condition:

(*) If $L$ is a lattice having $\mathbb{P}$, then there exist $a<b$ in $L$ and $c_{1}, c_{2} \in b / a$ with $c_{1} \vee c_{2}=b, c_{1} \wedge c_{2}=a, c_{1} \neq a$, and the lattice $c_{2} / a$ again having the property $\mathbb{P}$.

Then, any $L \in \mathcal{M} \cap \mathcal{U}$ having $\mathbb{P}$ is not $\mathrm{QFD}$.

We are now going to characterize QFD compactly generated modular lattices, which generalizes a result on QFD modules due to Camillo [17].

Definition 5.3. Let $L$ be a lattice. We say that a lattice $L$ verifies Condition $(C)$ (or Camillo's Condition) if for every $m \in L$ there exists a compact element $t$ of $L$ such that $t \leqslant m$ and $[t, m[$ has no maximal element.

The next result, whose proof essentially uses the Lemonnier's Lemma, is the latticial version of $[17$, Theorem]. 
TheOREM 5.4 ([4, Theorem 2.8]). A modular, compactly generated lattice $L$ is QFD if and only if $L$ verifies Condition $(C)$.

For arbitrary upper continuous modular lattices, we have the following characterization of the QFD condition.

THEOREM 5.5 ([4, Theorem 4.1]). A modular upper continuous lattice $L$ is QFD if and only if for every directed set $D \subseteq L$ there exists $d_{0} \in D$ such that, for every $d \in D$ with $d \in\left[d_{0}, \bigvee D\left[, d\right.\right.$ is small in $(\bigvee D) / d_{0}$.

We say that a non-trivial poset $P$ is dense if for any $a, b \in P$ with $a<b$ there exists $c \in P$ such that $a<c<b$.

THEOREM 5.6 ([4, Theorem 4.8]). Let L be an upper continuous modular lattice and denote $\varphi(x)=\bigvee\{k \mid k \in x / 0$, $k$ compact in $L\}, x \in L$. Assume that for every $a \in L$ with $a>\varphi(a)$ the interval $a / \varphi(a)$ is a dense poset. Then, $L$ is QFD if and only if $L$ verifies Condition $(C)$ and does not contain a sublattice isomorphic to the lattice $[0,1]^{\mathbb{N}}$ considered with the componentwise order, where $[0,1] \subseteq \mathbb{R}$.

We end this section by presenting the following latticial extension of a nice characterization of QFD modules in terms of finite meet irreducible decompositions we recently learned from Patrick Smith in a private communication, Ankara, August 2008:

Proposition 5.7. A lattice $L \in \mathcal{M} \cap \mathcal{U}$ is QFD if and only every element $1 \neq x \in L$ can be written as a finite meet of irreducible elements of $L$.

6. FT $_{\alpha}$ and $\mathbf{F T}_{\alpha}^{0}$. Taking into account that a poset $P$ is Artinian if and only if $k(P) \leqslant 0$, a part of Theorem 3.1 can be reformulated as follows:

$$
k\left(M_{R}\right) \leqslant 0 \Longleftrightarrow M \text { is QFD and } k(\mathcal{C}(M)) \leqslant 0 \Longleftrightarrow M \text { is QFD and } k(\mathcal{F}(M)) \leqslant 0 .
$$

Here, $k(\mathcal{C}(M))$ (resp. $k(\mathcal{F}(M))$ ) means the Krull dimension of the poset $\mathcal{C}(M)$ (resp. $\mathcal{F}(M)$ ) of all cyclic (resp. finitely generated) submodules of $M$, ordered by inclusion. This reformulation suggested the following extension for an arbitrary ordinal $\alpha$ :

Theorem 6.1 (The Dual $\alpha$-FAith Theorem FT $_{\alpha}^{0}$ ) ([8, Theorem 1.17]). For any $M_{R}$ and any ordinal $\alpha \geqslant 0$

$$
k\left(M_{R}\right) \leqslant \alpha \Longleftrightarrow M \text { is QFD and } k(\mathcal{F}(M)) \leqslant 0 .
$$

Because the compact elements of the lattice $\mathcal{L}(M)$ of all submodules of a module $M_{R}$ are exactly the finitely generated submodules of $M$, it is natural to ask whether a latticial extension of Theorem 6.1 is true; see Problem 8.2.

We are now going to discuss the validity of the Latticial $\alpha$-Faith Theorem FT $_{\alpha}$, that is,

$$
\text { FT }_{\alpha}: k^{0}(L) \leqslant \alpha \Longleftrightarrow L \text { is a QFD lattice and } k^{0}(\mathcal{S}(L)) \leqslant \alpha \text {, }
$$

for an arbitrary lattice $L \in \mathcal{M} \cap \mathcal{U}$ and an arbitrary ordinal $\alpha \geqslant 0$, where $\mathcal{S}(L)$ is the set of all subdirectly elements of $L$, also called CI elements of $L$.

An essential tool in establishing our results is the following extension to posets of the dual of a result due to Goodearl and Zimmermann-Huisgen [25] concerning the relationship between the Krull dimension of a module and the length of reverse well-ordered chains of its submodules. 
THEOREM 6.2 ([5, Theorem 1.11]). The following conditions are equivalent for a poset $P$.

(a) $k^{0}(P)$ exists and is countable.

(b) $\lambda(P)$ is countable.

In case conditions (a) and (b) are met and $k^{0}(P)=\alpha>0$, then

$$
\omega^{\beta}<\lambda(P) \leqslant \omega^{\alpha+1} \quad \text { for all ordinals } \beta<\alpha .
$$

In case $\alpha$ is finite, the lower bound for $\lambda(P)$ can be improved to $\omega^{\alpha}<\lambda(P)$.

For a poset $P$ we have denoted by $\lambda(P)$ the so called codepth of $P$; i.e. the least ordinal that does not embed in $P$. See also [30], where the term of depth of $P$, denoted by $\delta(P)$, has been defined as the least ordinal that does not embed in $P^{0}$. We have also denoted by $\omega$ the first transfinite ordinal, which is the order type of the set $\mathbb{N}=\{0,1,2, \ldots\}$ of natural numbers. For basic properties of the arithmetic of ordinal numbers, the reader is referred to [37].

Note that for any module $M_{R}$, the lattice $\mathcal{L}(M)$ of all submodules of $M$ has the property that for each $N<P$ in $\mathcal{L}(M)$, the quotient module $P / N$ has a subdirectly quotient module $P / Q$, so we may say that the lattice $\mathcal{L}(M)$ is 'rich in subdirectly irreducibles'. We take this property as definition for an arbitrary lattice or poset.

DEFINITION 6.3. A lattice $L$ is said to be rich in subdirectly irreducibles, abbreviated $R S I$, if for every $a<b$ in $L$, the interval $b / a$ has a subdirectly irreducible quotient interval $b / c \subseteq b / a$.

The property of a lattice $L$ being RSI is related to the property of $L$ being a lattice with completely irreducible decomposition, which means that every $1 \neq a \in L$ can be written as a meet of a family, not necessarily finite, of CI elements of $L$ (see [2, Remarks $0.15])$. Other recent results on completely irreducible submodules and their connections with primal submodules, primary submodules and their meet decompositions may be found in [12], [13].

The next result characterizes RSI lattices in terms of Gabriel dimension, so providing large classes of such lattices.

Proposition 6.4 ([5, Proposition 1.2]). A lattice $L \in \mathcal{M} \cap \mathcal{U}$ is RSI if and only if for each $a<b$ in L there exist $x<y$ in $b / a$ such that $y / x$ has Gabriel dimension. So, if $L$ has Gabriel dimension, then $L$ is RSI. In particular, if $L$ is Artinian, semi-Artinian, Noetherian, or has (dual) Krull dimension, then $L$ is RSI.

Now, we are going to show that the Latticial $\mathbf{F T}_{\alpha}$ holds for any finite ordinal $\alpha$ and for any upper continuous modular lattice $L$ which is RSI. In doing so, we have to characterize the existence and magnitude of $k^{0}(L)$ in terms of the existence and size of $k^{0}(\mathcal{S}(L))$. This task is achieved by the following very technical Lemma.

LeMma 6.5 ([5, Lemmas 1.13 and 1.17]). Let $L \in \mathcal{M} \cap \mathcal{U}$ be a QFD lattice which is RSI. Then, the following assertions hold.

(1) If $L$ contains a chain of order type $\omega^{\alpha}$ for some ordinal $\alpha \geqslant 1$, then $\mathcal{S}(L)$ contains a chain of order type $\omega^{\alpha}$.

(2) If $\mathcal{S}(L)$ has countable dual Krull dimension, then L has (dual) Krull dimension.

Theorem 6.6 ([5, Theorem 1.15]). Let $L \in \mathcal{M} \cap \mathcal{U}$, be such that $k^{0}(L)=\alpha$ is a countable ordinal. 
(1) If $1 \leqslant \alpha<\omega$ or if $\alpha$ is a limit ordinal, then $k^{0}(\mathcal{S}(L))=\alpha$.

(2) If $\alpha=\delta+1$ for some $\delta \geqslant-1$, then $k^{0}(\mathcal{S}(L))=\delta$ or $k^{0}(\mathcal{S}(L))=\delta+1$.

Proof. Clearly $k^{0}(\mathcal{S}(L))$ exists and $k^{0}(\mathcal{S}(L))=\gamma \leqslant \alpha$ for some ordinal $\gamma$. The conclusions of the theorem are trivial for $\alpha=0$; so we assume that $\alpha \geqslant 1$. By Theorem 6.2, we have $\lambda(\mathcal{S}(L)) \leqslant \omega^{\gamma+1} \leqslant \omega^{\alpha+1}, \omega^{\beta}<\lambda(L)$ for each $\beta<\alpha$, and $\omega^{\alpha}<\lambda(L)$ if $\alpha$ is finite. Thus $L$ contains a chain of type $\omega^{\beta}$ for each $\beta<\alpha$ and a chain of type $\omega^{\alpha}$ if $\alpha$ is finite. By Lemma $6.5(1), \mathcal{S}(L)$ also has chains of these types. Hence $\omega^{\beta}<\lambda(\mathcal{S}(L)) \leqslant \omega^{\gamma+1} \leqslant \omega^{\alpha+1}$ for each $\beta<\alpha$, and $\omega^{\alpha}<\lambda(\mathcal{S}(L))$ if $\alpha$ is finite. Now, (1) and (2) follow easily.

THEOREM 6.7 ([5, Theeorem 1.18]). The following conditions are equivalent for $L \in \mathcal{M} \cap \mathcal{U}$.

(1) $k^{0}(L)$ exists and is countable.

(2) $L$ is both QFD and RSI, and $k^{0}(\mathcal{S}(L))$ exists and is countable.

If conditions (1) or (2) are met and $-1 \leqslant k^{0}(\mathcal{S}(L))=\alpha$, then $k^{0}(L)=\alpha$ or $k^{0}(L)=$ $\alpha+1$. Moreover, $k^{0}(L)=\alpha$ if $0 \leqslant \alpha<\omega$.

Proof. (1) $\Longrightarrow$ (2) is trivial, and (2) $\Longrightarrow$ (1) follows by combining Lemma 6.5 and Theorem 6.2. The final assertions are immediate from Theorem 6.6.

Corollary 6.8 (The Latticial FT $_{n}$ ). For any $L \in \mathcal{M} \cap \mathcal{U}$ and $n \in \mathbb{N}$,

$$
k^{0}(L) \leqslant n \Longleftrightarrow L \text { is both QFD and RSI, and } k^{0}(\mathcal{S}(L)) \leqslant n .
$$

Since the lattice $\mathcal{L}\left(M_{R}\right)$ is always RSI, we obtain at once the $\mathbf{F T}_{n}$ for any module and any $n \in \mathbb{N}$ :

CoROllary $6.9\left(\mathbf{F T}_{n}\right)\left(\left[\mathbf{5}\right.\right.$, Corollary 1.19]). Let $M_{R}$ be a module, and let $n \in \mathbb{N}$. Then

$$
k^{0}\left(M_{R}\right) \leqslant n \Longleftrightarrow M_{R} \text { is QFD and } k^{0}\left(\mathcal{S}\left(M_{R}\right)\right) \leqslant n .
$$

In particular, for $n=0$, Corollary 6.9 gives precisely the Faith's Theorem FT.

The next result provides an evaluation of the dual Krull dimension $k^{0}(L)$ of a lattice $L$ in terms of irreducible and completely irreducibles elements of $L$. Note that the proof of $(1) \Longleftrightarrow(2)$ in theorem below is based on two main ingredients: the Lemonnier's Lemma (see Lemma 5.2) and a corrected version of [10, Proposition 3.10] (see [11]) involving the subclass

$$
\rangle \mathcal{K}_{\alpha}^{0} \ll:=\left\{X \in \mathcal{M} \mid \forall a<b \text { in } X, \exists c \in\left[a, b\left[, k^{0}(c / a) \leqslant \alpha\right\}\right.\right.
$$

of the class $\mathcal{M}$ of all modular lattices with 0 and 1 .

THEOREM 6.10 ([5, Theorems 1.21 and 1.22]). The following statements are equivalent for an arbitrary ordinal $\alpha \geqslant 0$ and a lattice $L \in \mathcal{M} \cap \mathcal{U}$.

(1) $k^{0}(L) \leqslant \alpha$.

(2) $L$ is QFD, $L$ is RSI, and $k^{0}(1 / x) \leqslant \alpha$ for all $x \in \mathcal{S}(L)$.

(3) $L$ is QFD and $k^{0}(1 / x) \leqslant \alpha$ for all $x \in \mathcal{I}(L)$.

COROLlARY 6.11 ([5, Corollary 1.24]). The following statements are equivalent for a module $M_{R}$ and an arbitrary ordinal $\alpha \geqslant 0$. 
(1) $k^{0}\left(M_{R}\right) \leqslant \alpha$.

(2) $M_{R}$ is QFD and $k^{0}(M / N) \leqslant \alpha$ for every (completely) irreducible submodule $N$ of $M$.

Using Theorem 5.5 we obtain the following evaluation of $k^{0}(L)$ in terms of small elements of $L$.

THEOREM 6.12 ([5, Theorem 1.28]). The following statements are equivalent for a lattice $L \in \mathcal{M} \cap \mathcal{U}$ and an arbitrary ordinal $\alpha \geqslant 0$.

(1) $k^{0}(L) \leqslant \alpha$.

(2) For every $x \in L$, there exists $s \leqslant x$ such that $s$ is small in $x / 0$ and $k^{0}(x / s) \leqslant$ $\alpha$.

An immediate consequence of Theorem 6.12 is the following dual of a result due to Huynh, Dung and Smith [29, Lemma 6] (see also [19, Theorem 6.3]) which answers in the positive an Open Problem raised by Albu and Rizvi [8, p. 1923]:

COROLlary 6.13 ([5, Corollary 1.29]). The following statements are equivalent for a module $M_{R}$ and an ordinal $\alpha \geqslant 0$.

(1) $k^{0}(M) \leqslant \alpha$.

(2) Every submodule $X$ of $M$ has a small submodule $S$ with $k^{0}(X / S) \leqslant \alpha$.

7. Applications to torsion theories and Grothendieck categories. Throughout this section $\tau=(\mathcal{T}, \mathcal{F})$ will be a fixed hereditary torsion theory on Mod- $R$. The set $F_{\tau}:=$ $\left\{I \leqslant R_{R} \mid R / I \in \mathcal{T}\right\}$ is called the Gabriel topology associated with $\tau$. For any $M_{R}$ we denote $\operatorname{Sat}_{\tau}(M)=\{N \mid N \leqslant M, M / N \in \mathcal{F}\}$, and for any $N \leqslant M$ we denote by $\bar{N}=\bigcap\{C \mid N \leqslant C \leqslant M, M / C \in \mathcal{F}\}$ the $\tau$-closure of $N$ in $M$. It is known that $\operatorname{Sat}_{\tau}(M)$ is an upper continuous modular lattice for any $M_{R}$ (see [39, Chapter 9, Proposition 4.1]).

As in $[\mathbf{4}, \mathbf{5}]$, a module $M_{R}$ is said to be $\tau$-QFD if the lattice $\operatorname{Sat}_{\tau}(M)$ is QFD. More generally, if $\mathbb{P}$ is any property on lattices, we say that a module $M_{R}$ is/has $\tau-\mathbb{P}$ if the lattice $\operatorname{Sat}_{\tau}(M)$ is/has $\mathbb{P}$. Thus, we obtain the concepts of a $\tau$-Artinian module, $\tau$-Noetherian module, $\tau$-RSI module, etc. The $\tau$-Krull dimension $k_{\tau}(M)$ (resp. $\tau$-dual Krull dimension $k_{\tau}^{0}(M)$ ) of $M$ is defined as the Krull dimension (resp. dual Krull dimension) of the lattice $\operatorname{Sat}_{\tau}(M)$.

For all undefined notation and terminology on torsion theories the reader is referred to [7, 24] and/or [39].

An important problem in Module Theory appeared about 40 years ago is to relativize a certain property, that is,

$$
\begin{gathered}
\text { Given a property } \mathbb{P} \text { in the lattice } \mathcal{L}\left(M_{R}\right) \text {, investigate the property } \mathbb{P} \\
\text { in the lattice } \operatorname{Sat}_{\tau}\left(M_{R}\right) ;
\end{gathered}
$$

in other words,

Having a theorem $\mathbb{T}$ on modules, investigate its relativization $\tau-\mathbb{T}$.

The best illustration of this problem is the relativization of the renowned HopkinsLevitzki Theorem, abbreviated H-LT:

H-LT: Any right Artinian ring $R$ with identity is right Noetherian. 
The relativization of this theorem with respect to a hereditary torsion theory $\tau$ on Mod-R:

$\tau$-H-LT: Any right $\tau$-Artinian ring $R$ with identity is right $\tau$-Noetherian,

is also known as the Teply-Miller Theorem.

Let us mention that the module-theoretical proofs available in the literature of the Relative H-LT ( $\tau$-H-LT), namely the original one in 1979 due to Miller and Teply [34, Theorem 1.4], and another one in 1982 due to Faith [20, Theorem 7.1 and Corollary 7.2], are very long and complicated; so, the relativization of a result on modules is not always a simple job, and sometimes it may be even impossible. A very simple and natural approach to the $\tau$-H-LT is to formulate and prove it in the most general latticial setting of an arbitrary modular lattice with 0 and 1, and then to apply it for the lattice $\operatorname{Sat}_{\tau}\left(R_{R}\right)$. This has been done by Albu and Smith [9, Theorem 1.9]. For a very thorough discussion on the various aspects of the H-LT and the connections between them, see the survey paper [3].

As we have already seen, our characterizations of upper continuous modular lattices $L$ with $k^{0}(L) \leqslant \alpha$ require the lattice $L$ to be RSI. This condition is automatically satisfied for the lattice of submodules of any module $M$. But, for an arbitrary hereditary torsion theory $\tau$ on Mod- $R$, the lattice $\operatorname{Sat}_{\tau}(M)$ may fail to be RSI. Therefore, we first look for sufficient conditions on $\tau$ to insure that, for any module $M_{R}$, the lattice $\operatorname{Sat}_{\tau}(M)$ is RSI, i.e. any module $M_{R}$ is $\tau$-RSI.

We denote by $\operatorname{Max}_{\tau}(R)$ the set of all maximal elements of the poset

$$
\left(\operatorname{Sat}_{\tau}\left(R_{R}\right) \backslash\{R\}, \subseteq\right) .
$$

Note that we may have $\operatorname{Max}_{\tau}(R)=\varnothing$ (see, e.g. [6, Remarques 2.5 (2)]). However, if the Gabriel topology $F_{\tau}$ has a basis of finitely generated right ideals, then, the poset $\left(\operatorname{Sat}_{\tau}\left(R_{R}\right) \backslash\{R\}, \subseteq\right)$ is inductive, and so, a $\tau$-relative Krull Lemma holds:

$$
\forall I \in \operatorname{Sat}_{\tau}\left(R_{R}\right) \backslash\{R\}, \exists J \in \operatorname{Max}_{\tau}(R) \text { such that } I \subseteq J,
$$

and, in particular, we have $\operatorname{Max}_{\tau}(R) \neq \varnothing$. Recall that by a basis of the Gabriel topology $F_{\tau}$ we mean a subset $B$ of $F_{\tau}$ such that every right ideal in $F_{\tau}$ contains some $J \in B$. For such torsion theories $\tau$ satisfying the condition ( $\dagger$ ), any module $M_{R}$ is $\tau$-RSI by [5, Proposition 2.5]. On the contrary, if $\operatorname{Max}_{\tau}(R)=\varnothing$, then the module $R_{R}$ is not $\tau$-RSI.

The latticial results from the previous sections can be now easily specialized from an arbitrary upper continuous modular lattice $L$ to lattices of type $\operatorname{Sat}_{\tau}\left(M_{R}\right)$. We present below only three of them, and leave to the reader the pleasure to do it for the remaining ones.

THEOREM 7.1 ( $\tau$-FT) ([5, Corollary 2.9]). Assume that the Gabriel topology $F_{\tau}$ for the hereditary torsion theory $\tau$ has a basis of finitely generated right ideals. Then the following assertions are equivalent for a module $M_{R}$.

(1) $M$ is $\tau$-Noetherian.

(1) $M$ is $\tau$-QFD and $\operatorname{Sat}_{\tau}\left(M_{R}\right)$ has ACC on its subdirectly irreducible elements.

COROLlaRY 7.2 ([5, Corollary 2.10]). A ring $R$ is right $\tau$-Noetherian if and only if the following conditions are satisfied.

(a) $R_{R}$ is $\tau$-QFD;

(b) $\operatorname{Sat}_{\tau}\left(R_{R}\right)$ has ACC on its subdirectly irreducible elements;

(c) the Gabriel topology $F_{\tau}$ has a basis of finitely generated right ideals. 
THEOREM $7.3\left(\tau-\mathbf{F T}_{n}\right)$. Let $\tau$ be a hereditary torsion theory on Mod- $R$ satisfying the $\tau$-Krull Lemma condition ( $\dagger$ ). Then, for any module $M_{R}$ and any $n \in \mathbb{N}$ we have:

$$
k_{\tau}^{0}\left(M_{R}\right) \leqslant n \Longleftrightarrow M_{R} \text { is } \tau \text {-QFD and } k^{0}\left(\mathcal{S}\left(\operatorname{Sat}_{\tau}(M)\right) \leqslant n .\right.
$$

For the remaining of this section $\mathcal{G}$ will denote a fixed Grothendieck category, that is, an Abelian category with exact direct limits and with a generator. For any object $X \in \mathcal{G}, \mathcal{L}(X)$ will denote the lattice of all subobjects of $X$. It is well known that $\mathcal{L}(X)$ is an upper continuous modular lattice (see e.g. [39, Chapter 4, Proposition 5.3, and Chapter 5, Section 1]. For all undefined notation and terminology on Abelian categories the reader is referred to [7] and/or [39].

We say that an object $X \in \mathcal{G}$ is subdirectly irreducible, abbreviated SI, if the lattice $\mathcal{L}(X)$ is subdirectly irreducible. More generally, if $\mathbb{P}$ is any property on lattices, we say that an object $X \in \mathcal{G}$ is/has $\mathbb{P}$ if the lattice $\mathcal{L}(X)$ is/has $\mathbb{P}$. Thus, we obtain the concepts of co-irreducible (uniform) object, object rich in subdirectly irreducibles (RSI), object of finite Goldie dimension, object with (dual) Krull dimension, QFD object, etc. If $X$ has Krull dimension (resp. dual Krull dimension), we write $k(X):=k(\mathcal{L}(X))$ (resp. $k^{0}(X):=k^{0}(\mathcal{L}(X))$. Similarly, a subobject $Y$ of an object $X \in \mathcal{G}$ is/has $\mathbb{P}$ if the element $Y$ of the lattice $\mathcal{L}(X)$ is/has $\mathbb{P}$. We denote by

$$
\mathcal{S}(X):=\mathcal{S}(\mathcal{L}(X))=\{Y \leqslant X \mid X / Y \text { is SI }\}
$$

the set of all CI subobjects of an object $X \in \mathcal{G}$, which were called 'subdirectly irreducible subobjects' of $X$ in [5].

The existence of CI subobjects of an object $X \in \mathcal{G}$ is intimately related to the existence of simple objects of $\mathcal{G}$. It may happen that $\mathcal{G}$ has no simple object (see e.g. [14, p. 1539]). For such a category $\mathcal{G}$, the only object in $\mathcal{G}$ having (dual) Krull dimension is the zero object of $\mathcal{G}$, and no non-zero object of $\mathcal{G}$ is RSI.

The next result characterizes those Grothendieck categories $\mathcal{G}$ having a finitely generated generator. Recall that an object $C \in \mathcal{G}$ is called finitely generated if $C$ is a compact element of the lattice $\mathcal{L}(C)$ of all subobjects of $C$.

Proposition 7.4 ([5, Proposition 2.12]). The following assertions are equivalent for a Grothendieck category $\mathcal{G}$.

(1) $\mathcal{G}$ has a finitely generated generator.

(2) There exists a unital ring $A$ and a hereditary torsion theory $\chi=(\mathcal{H}, \mathcal{E})$ on Mod- $A$ such that $\mathcal{G} \simeq \operatorname{Mod}-A / \mathcal{H}$ and the Gabriel topology $F_{\chi}$ has a basis of finitely generated right ideals of $A$.

By Proposition 7.4, any Grothendieck category $\mathcal{G}$ having a finitely generated generator has simple objects, and any $X \in \mathcal{G}$ is RSI. A recent result of Albu and Van Den Berg [14, p. 1545]) provides an example of an indecomposable non-locally finitely generated Grothendieck category with a single simple object, and answers in the negative a sharper form of Question 2.14 raised by Albu et al. [5] asking whether a Grothendieck category having simple objects has a finitely generated generator.

We end this paper by presenting specializations of a few latticial results of the previous sections from an arbitrary upper continuous modular lattice $L$ to lattices of type $\mathcal{L}(X), X$ object of a Grothendieck category $\mathcal{G}$ having a finitely generated generator. As observed just after Proposition 7.4, any object of such a category is RSI, so the three results below are immediate consequences of the corresponding latticial results. Notice that there is a slight change of terminology in the next two results 
when comparing them with the original ones of [5]: instead of the term of 'subdirectly irreducible subobject' we use the more appropriate term of 'completely irreducible subobject', abbreviated CI.

THEOREM 7.5 ([5, Corollary 2.18]). The following assertions are equivalent for a Grothendieck category $\mathcal{G}$ having a finitely generated generator, an object $X \in \mathcal{G}$, and an ordinal $\alpha \geqslant 0$.

(1) $k^{0}(X) \leqslant \alpha$.

(2) $X$ is QFD and $k^{0}(X / Y) \leqslant \alpha$ for every (completely) irreducible subobject $Y$ of $X$.

THEOREM 7.6 (CATEGORICAL FT) ([5, Corollary 2.19]). The following assertions are equivalent for a Grothendieck category $\mathcal{G}$ having a finitely generated generator and an object $X \in \mathcal{G}$.

(1) $X$ is Noetherian.

(2) $X$ is QFD and has ACC on its CI subobjects.

THEOREM 7.7 (CATEGORICAL FT ${ }_{n}$ ). Let $\mathcal{G}$ be a Grothendieck category having a finitely generated generator. Then, for any object $X \in \mathcal{G}$ and any $n \in \mathbb{N}$ we have:

$$
k^{0}(X) \leqslant n \Longleftrightarrow X \text { is QFD and } k^{0}(\mathcal{S}(X)) \leqslant n .
$$

\section{Some open questions.}

Problem 8.1. Can $\mathcal{F}(M)$ be replaced in Theorem 6.1 by its subset $\mathcal{C}(M)$ ?

Problem 8.2 (The Latticial FT $_{\alpha}^{0}$ ). Let $L \in \mathcal{M} \cap \mathcal{U}$, and denote by $\mathcal{C}(L)$ the set of all compact elements of $L$. Then

$$
k(L) \leqslant \alpha \Longleftrightarrow L \text { is QFD and } k(\mathcal{C}(L)) \leqslant \alpha .
$$

In particular, $L$ is Artinian $\Longleftrightarrow L$ is QFD and $\mathcal{C}(L)$ is an Artinian poset.

Problem 8.3 (The Latticial FT $_{\alpha}$ ). For any $L \in \mathcal{M} \cap \mathcal{U}$ and any ordinal $\alpha \geqslant 0$,

$$
k^{0}(L) \leqslant \alpha \Longleftrightarrow L \text { is both QFD and RSI, and } k^{0}(\mathcal{S}(L)) \leqslant \alpha .
$$

Problem 8.4. Do dual characterizations to those in Theorem 6.10 hold for $L \in$ $\mathcal{M} \cap \mathcal{U}$ having $k(L) \leqslant \alpha$ ?

ACKNOWLEDGEMENTS. The author thanks the referee for his/her careful reading of the manuscript and helpful suggestions. The author gratefully acknowledges partial financial support from the grant ID - PCE 1190/2008 awarded by the Consiliul Naţional al Cercetării Ştiinţifice în Învăţământul Superior (CNCSIS), România.

\section{REFERENCES}

1. T. Albu, Gabriel dimension of partially ordered sets (I), Bull. Math. Soc. Sci. Math. Roumanie 28(76) (1984), 99-108.

2. T. Albu, Completely irreducible meet decompositions in lattices, with applications to Grothendieck categories and torsion theories (I), Bull. Math. Soc. Sci. Math. Roumanie 52(100) (2009), 393-419. 
3. T. Albu, A seventy years jubilee: The Hopkins-Levitzki Theorem, in Ring and module theory, trends in mathematics (Albu T., Birkenmeier G. F., Erdoğan A. and Tercan A., Editors) (Birkhäuser, Basel, 2010), 1-26.

4. T. Albu, M. Iosif and M. L. Teply, Modular QFD lattices with applications to Grothendieck categories and torsion theories, J. Algebra Appl. 3 (2004), 391-410.

5. T. Albu, M. Iosif and M. L. Teply, Dual Krull dimension and quotient finite dimensionality, J. Algebra 284 (2005), 52-79.

6. T. Albu and C. Năstăsescu, Décompositions primaires dans les catégories de Grothendieck commutatives (I), J. Reine Angew. Math. 280 (1976), 172-194.

7. T. Albu and C. Năstăsescu, Relative finiteness in module theory (Marcel Dekker, Inc., New York and Basel, 1984).

8. T. Albu and S. T. Rizvi, Chain conditions on quotient finite dimensional modules, Commun. Algebra 29 (2001), 1909-1928.

9. T. Albu and P. F. Smith, Localization of modular lattices, Krull dimension, and the Hopkins-Levitzki Theorem (I), Math. Proc. Camb. Phil. Soc. 120 (1996), 87-101.

10. T. Albu and P. F. Smith, Localization of modular lattices, Krull dimension, and the Hopkins-Levitzki Theorem (II), Commun. Algebra 25 (1997), 1111-1128.

11. T. Albu and P. F. Smith, Corrigendum and addendum to 'Localization of Modular Lattices, Krull dimension, and the Hopkins-Levitzki Theorem (II)', Commun. Algebra 29 (2001), 3677-3682.

12. T. Albu and P. F. Smith, Primal, completely irreducible, and primary meet decompositions in modules, Preprint Series of the Institute of Mathematics of the Romanian Academy, Preprint nr. $1 / 2009$.

13. T. Albu and P. F. Smith, Primality, irreducibility, and complete irreducibility in modules over commutative rings, Rev. Roumaine Math. Pures Appl. 54 (2009), 275-286.

14. T. Albu and J. Van Den Berg, An indecomposable non-locally finitely generated Grothendieck category with simple objects, J. Algebra 321 (2009), 1538-1545.

15. G. Birkhoff, Subdirect unions in universal algebra, Bull. Amer. Math. Soc. 50 (1944), 764-768.

16. G. Birkhoff, Lattice theory, 3rd ed. (American Mathematical Society, Providence, RI, 1967).

17. V. P. Camillo, Modules whose quotients have finite Goldie Dimension, Pacific J. Math. 69 (1977), 337-338.

18. P. Crawley and R. P. Dilworth, Algebraic theory of lattices, (Prentice-Hall, Englewood Cliffs, NJ, 1973).

19. N. V. Dung, D. V. Huynh, P. F. Smith and R. Wisbauer, Extending modules (Longman, Harlow, 1990).

20. C. Faith, Injective modules and injective quotient rings, Lecture Notes in Pure and Applied Mathematics (Marcel Dekker, Inc., New York and Basel, 1982).

21. C. Faith, Quotient finite dimensional modules with ACC on subdirectly irreducible submodules are Noetherian, Commun. Algebra 27 (1999), 1807-1810.

22. C. Faith, Rings and things and a fine array of twentieth century associative algebra, 2nd ed. (American Mathematical Society, Providence, RI, 2004).

23. L. Fuchs, Infinite Abelian groups, vol. I (Academic Press, New York and London, 1970).

24. J. S. Golan, Torsion theories (Pitman/Longman, New York, 1986).

25. K. Goodearl and B. Zimmermann-Huisgen, Lengths of submodule chains versus Krull dimension in non-Noetherian rings, Math. Z. 191 (1986), 519-527.

26. G. Grätzer, General lattice theory, 2nd ed. (Birkhäuser Verlag, Basel Boston Berlin, 2003).

27. P. Grzeszczuk and E. R. Puczyłowski, On Goldie and dual Goldie dimension, J. Pure Appl. Algebra 31 (1984), 47-54.

28. D. Herbera and A. Shamsuddin, Modules with semi-local endomorphism ring, Proc. Amer. Math. Soc. 123 (1995), 3593-3600.

29. D. V. Huynh, N. V. Dung and P. F. Smith, A characterization of rings with Krull dimension, J. Algebra 32 (1990), 104-112.

30. W. G. Lau, M. L. Teply and A. K. Boyle, The deviation, density, and depth of partially ordered sets, J. Pure Appl. Algebra 60 (1989), 253-268. 
31. B. Lemonnier, Déviation des ensembles et groupes abéliens totalement ordonnés, Bull. Sci. Math. $2^{e}$ série 96 (1972), 289-303.

32. B. Lemonnier, Dimension de Krull et codéviation. Application au théorème d'Eakin, Commun. Algebra 16 (1978), 1647-1665.

33. J. C. McConnell and J. C. Robson, Noncommutative noetherian rings (John Wiley \& Sons, Chichester, New York, Brisbane, Toronto, Singapore, 1987).

34. R. W. Miller and M. L. Teply, The descending chain condition relative to a torsion theory, Pacific J. Math. 83 (1979), 207-220.

35. C. Năstăsescu and F. Van Oystaeyen, Dimensions of ring theory (D. Reidel Publishing Company, Dordrecht, Boston, Lancaster, Tokyo, 1987).

36. E. R. Puczyłowski, Linear properties of Goldie dimension of modules and modular lattices, Glasgow Math. J. 52A (2010).

37. J. G. Rosenstein, Linear orderings (Academic Press, New York, 1982).

38. B. Sarath and K. Varadarajan, Dual Goldie dimension - II, Commun. Algebra 7 (1979), $1885-1899$.

39. B. Stenström, Rings of quotients (Springer-Verlag, Berlin, Heidelberg, New York, 1975).

40. K. Varadarajan, Dual Goldie dimension, Commun. Algebra 7 (1979), 565-510. $83-92$.

41. K. Varadarajan, Properties of endomorphism rings, Acta Math. Hungar. 74 (1997),

42. R. Wisbauer, Foundations of module and ting theory (Gordon and Breach Science Publishers, Philadelphia, Reading, Paris, Tokyo, Melbourne, 1991). 